\title{
Sulcal Segmentation for Cortical Thickness Measurements
}

\author{
Chloe Hutton, Enrico De Vita, and Robert Turner
}

The Wellcome Department of Imaging Neuroscience, 12 Queen Square, ION, UCL, London WC1N 3BG, UK

\{chutton, edevita,rturner\}@fil.ion.ucl.ac.uk

http://www.fil.ion.ucl.ac.uk

\begin{abstract}
Thickness of the cerebral cortex may provide valuable information about normal and abnormal neuroanatomy. For accurate cortical thickness measurements in brain MRI, precise segmentation of the grey matter border is necessary. In this paper we specifically address the problem of extracting the deep cortical folds or sulci, which can be difficult to resolve or totally obscured due to limited MRI resolution and contrast. We propose a method that iteratively solves Laplace's equation for adjacent sub-layers of the cortex. This approach preserves the laminar structure of the cortex and provides clear definition of deep sulci. The implementation is computationally efficient. We present inter-subject and intra-subject results that are consistent with the literature.
\end{abstract}

\section{Introduction}

The human cortex is comprised of a sheet of grey matter surrounding white matter. The thickness of the grey matter sheet is of great interest in studies of normal and abnormal neuroanatomy. Within individual brains cortical thickness varies from region to region, reflecting changes in the underlying cytoarchitecture. These changes relate to differences in cell types [1] and may also be associated with functionally distinct areas [2]. Although cortical thickness varies between individuals, abnormally thick or thin cortex may correlate with specific neuropathology and neurological conditions (e.g. [3, 4]) suggesting that thickness may be useful as a diagnostic tool.

Until recently, studies of cortical thickness have mostly involved postmortem measurements. With high resolution neuroanatomical MRI it is possible to make measurements in vivo. However, making these measurements manually necessitates displaying the brain as 2-D slices when in fact the cortex is a highly convoluted 3-D structure. Therefore accurate measurements of cortical thickness can only be made if the image plane is orthogonal to the cortical surface in the region of interest. As the direction of the cortical surface is constantly varying, manual measurements are susceptible to errors. Many methods have been proposed for segmenting brain MRI (e.g. [5]). In this paper we present a method that focuses on the segmentation of deep folds of grey matter (sulci) for the automated measurement of cortical thickness. Our approach divides the cortex into sub-layers of a specified thickness. We use Laplace's equation to calculate the thickness of adjacent layers and determine whether it is equal to the specified layer thickness. Sulci are identified where this equality breaks down. Our approach exploits the laminar structure of the cortex to segment sulci that 
cannot be identified in the MR image because of limited spatial resolution and contrast.

\section{Issues Related to Measuring Cortical Thickness}

A basic requirement for accurate brain morphometry is good quality, high contrast and resolution $\left(\bullet 1 \mathrm{~mm}^{3}\right)$ MRI. For cortical thickness measurements, the finest details of interest are the deep sulci between folds of grey matter that are surrounded by cerebrospinal fluid (CSF). In T1-weighted images, most commonly used for neuroanatomical investigations, grey and white matter have similar intensities so it is crucial to optimize the contrast between them for successful tissue segmentation [6]. CSF has a much lower intensity, but small CSF spaces can be difficult to resolve because of partial volume effects (where more than one tissue type co-exists in a voxel). The following sections highlight the main issues related to measuring cortical thickness.

Extraction of Grey Matter Boundaries: For measuring cortical thickness, the grey matter boundaries following deep sulcal folds must be accurately segmented. Methods have been proposed that fit deformable models to the cortical surface [7-10]. A disadvantage of such methods is the long processing times. Here, we present a computationally efficient method that extracts grey matter boundaries, segments deep sulci and calculates cortical thickness in less than an hour for $1 \mathrm{~mm}$ resolution whole brain images.

Segmenting Deep Sulci: If the narrow CSF spaces between sulci are not well resolved, regions of cortex can become 'hidden' and thickness measurements can be overestimated as illustrated in figure 1. The deformable model methods in [8] and [9] address this 'hidden cortex' problem using topological constraints that explicitly prevent points on the same surfaces from coming too close together and points on opposing surfaces from being too far apart. The disadvantage of this approach is that the long processing times required for deformable model methods are further increased by these additional constraints. In [10], sulcal locations are estimated using image averaging and subtraction to amplify regions with high frequency changes. The resulting decrease in CSF intensity is then identified using 'thinning' routines.

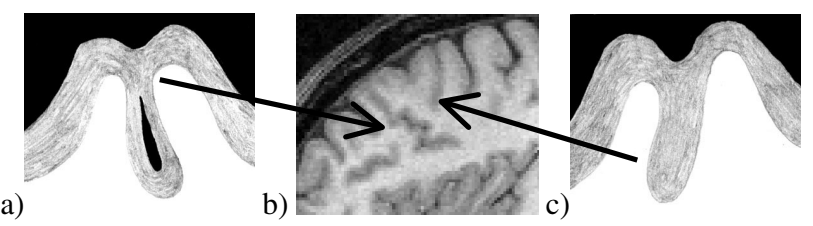

Fig. 1. 'Hidden cortex' where cortical thickness may be overestimated. a) Incorrectly resolved banks of cortex. b) Examples of a) and c) on MRI. c) Incorrectly resolved bottom of sulcus 
We address the 'hidden cortex' problem by modeling the cortical sheet as a series of adjacent sub-layers of a specified thickness. We then calculate the thickness of each sub-layer and compare it with the specified layer thickness. Sulcal locations are identified where the calculated thickness of a sub-layer is greater than the specified thickness. To calculate the thickness of each layer, we use the definition of thickness proposed in [10] and described in the following section.

Definition of Cortical Thickness as a Robust Metric: The thickness of the cortical sheet can be thought of as the distance between the two surfaces bordering the grey matter sheet. However, the cortical sheet is a highly folded three-dimensional structure with variable thickness so the definition of thickness as a metric must be carefully considered. For example, if thickness is defined as the straight-line distance between the two surfaces, this distance could be the perpendicular projection from one surface to the other (Fig. 2a) or the minimum distance from one surface to the other (Fig 2b). The problem with these straight-line definitions is that if the two surfaces are not parallel, the thickness will be different depending on which surface the measurement is made from (as illustrated in Figures $2 a$ and $b$ ). We therefore use the non-straight line definition of thickness outlined in [10]. This approach models the cortex as a series of nested sub-layers that are bounded by the inner and outer grey matter surfaces. The thickness at any point in the cortex is calculated by integrating along the direction perpendicular to each sub-layer between the two surfaces.

The sub-layers are modeled by assigning different boundary conditions to the two surfaces and solving Laplace's equation at each point between them. Laplace's equation is a second order partial differential equation for a scalar field $\Psi$ that can be enclosed between two boundaries and has the following form:

$$
\nabla^{2} \Psi=\frac{\partial^{2} \Psi}{\partial \mathrm{x}^{2}}+\frac{\partial^{2} \Psi}{\partial \mathrm{y}^{2}}+\frac{\partial^{2} \Psi}{\partial \mathrm{z}^{2}}=0
$$

Equation 1 can be solved iteratively using a standard relaxation method such as the one described in [10]. The resulting scalar field makes a smooth transition from one surface to the other describing the nested sub-layers (dashed lines in Fig. 2c). The perpendicular direction of each point in each sub-layer is determined by calculating the gradients of the scalar field which are normalized to produce a tangential vector field (solid lines with arrows in Fig. 2c). Integrating along the direction of the tangential vector field at any point in the cortex provides the cortical thickness.
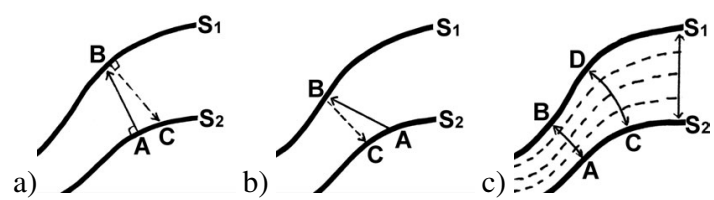

Fig. 2. Definitions of thickness between surfaces $S_{1}$ and $S_{2}$. a) The perpendicular projection from A to B - B to C. b) The minimum distance from A to B $-\mathrm{B}$ to C. c) Thickness defined using Laplace's equation (solid lines) from $\mathrm{A}$ to $\mathrm{B}$ and $\mathrm{C}$ to $\mathrm{D}$ 


\section{Sulcal Segmentation and Cortical Thickness Measurement}

\subsection{Tissue Segmentation and Preparation of Grey Matter Boundaries}

Initial tissue segmentation is carried out using the method implemented in SPM'99 [11], which combines tissue classification with correction for image inhomogeneities. For this work, the segmentation procedure was adapted to include a cutting plane through the brain stem separating cortical white matter from the cerebellum. The resulting probability maps of CSF, grey and white matter plus the cutting plane (matched with the image) are used in the following steps: 1) Label white matter when white matter probability is greater than that for grey matter. A 'connected component' analysis determines the final map of cortical white matter voxels (separated from the brain stem using the cutting plane). 2) Label grey matter when grey matter probability is greater than that for CSF. The largest grey matter connected component is selected as the initial map of grey matter voxels.

\subsection{Segmentation of Sulci}

Next, we must identify sulcal spaces that were incorrectly labeled as grey matter in the previous step. We do this by successively adding grey matter layers of an arbitrarily specified thickness to surround the white matter (Fig. 3a-b). Using Laplace's equation we calculate vector normals and thickness for the sub-layer. Voxels for which the calculated thickness is greater than the specified thickness are identified as belonging to sulci because they must be in contact with grey matter voxels within the same layer but from an opposing sulcal bank (Fig. 3c).

The initial grey matter map also includes the cerebellum and some extra-cortical tissue attached to cortical grey matter. We must therefore determine the limits of the cortical grey matter using image intensity (which is low for CSF) and intensity gradient information (high at the grey matter CSF boundary). This border prevents grey matter layers from going beyond the cortex.

The processing steps are illustrated in Fig. 3. and described below.

Repeat the following steps while there are voxels to process within the grey matter limits:

1: Add a layer of grey matter of specified thickness $T$ to surround previous layer. $\mathbf{1}^{\text {st }}$ layer: add layer to white matter in direction of pre-computed normals of white matter surface (Fig 3a).

Nth layer: add layer to previous layer in direction of normals of previous layer (Fig 3b).

2: Solve Laplace's equation for layer to determine the normal direction of each voxel.

3: Calculate the thickness at each voxel. Label voxels with a thickness $>\mathrm{T}$ as sulci (Fig 3c).

Sulcal voxels must be connected to the CSF voxels outside the cortex so that the grey matter sheet is bounded by two complete surfaces. This is achieved with the following morphological operations:

1: Combine CSF voxels with sulcal voxels and dilate the result.

2: Find the largest connected component of dilated CSF+sulci map (resulting from step 1).

3: Erode the CSF-sulci connected component.

4: Find largest connected component of the eroded CSF+sulci = final CSF+sulci (Fig 3d). 


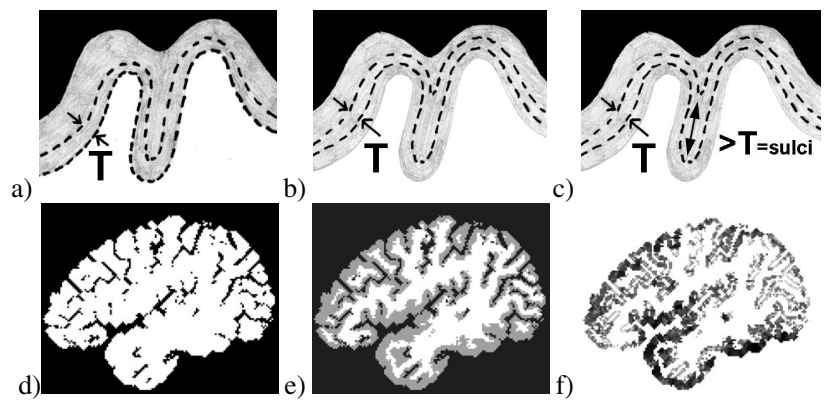

Fig. 3. Sulcal segmentation. a) $\mathbf{1}^{\text {st }}$ layer: add layer (dashed lines) of grey matter, thickness $\mathrm{T}$ to white matter. b) $\mathbf{N}^{\text {th }}$ layer: add layer (dashed lines) of grey matter, thickness $T$ to previous layer. c) Label voxels with thickness $>$ T as sulci. d) Map of CSF and sulci. e) Grey matter borders $\mathrm{f}$ ) Averaged thickness map (darker = greater thickness)

The final grey matter CSF border that includes the sulci (Fig 3d) is combined with the white matter surface so that two complete boundaries surround the grey matter (Fig 3e). Laplace's equation is solved for the resulting surfaces and the cortical thickness at each point is calculated using integration as described in [10] (Fig. 3f).

In this work we have used an arbitrary thickness of $\mathrm{T}=1 \mathrm{~mm}$, a $1 \mathrm{~mm}$ sampling size for solving Laplace's equation and for calculating thickness. However, using interpolation, the method can be adapted to use sub-voxel thickness and image sampling sizes. Averaged cortical thickness maps are calculated by replacing each thickness value with the mean of the thicknesses along each path between the two surfaces.

\subsection{Implementation and Image Acquisition}

The routines described above are implemented in Matlab 5 [12]. Using a pseudoparallel implementation, a full volumetric image of the brain can be processed in less than an hour on a standard desktop computer. Anatomical MRI of $1 \mathrm{~mm}^{3}$ resolution were acquired on a Siemens Vision 2T scanner with an MP-RAGE sequence optimised for grey and white matter contrast and minimal inhomogeneities [6].

\section{Results}

We applied the above methods to MR brain volumes of 3 subjects. For subject 1, 3 volumes were acquired during different scanning sessions. We calculated averaged cortical thickness maps and thickness histograms for each subject with and without sulcal segmentation. For subject 1, we matched the 3 images together using SPM'99, applied the resulting transformations to the thickness maps then calculated the mean and standard deviation of the averaged thickness maps. We generated isosurface renderings of the final thickness maps using Matlab 6 [12].

Identification of Deep Sulci. Figure 4 shows a slice from each of the three subjects with the border between grey matter and CSF overlaid in black. The grey scale of the images has been inverted and the borders thickened to improve visualization of the results. The original images are shown in the right column. The grey matter CSF 
border without sulcal segmentation is shown in the left column and with sulcal segmentation in the middle column. The figure clearly shows that many sulci, especially deep ones are only detected using the sulcal segmentation method.

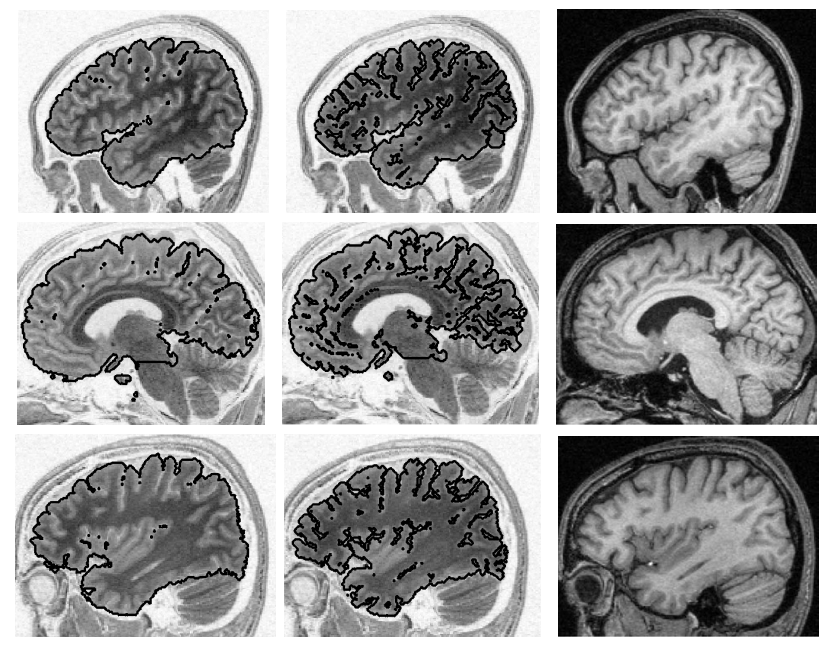

Fig. 4. Example slices from each subject overlaid with grey matter-CSF border without (left column) or with (middle column) sulcal segmentation. Original images shown in right column

Histograms of Averaged Cortical Thickness Maps. Figure 5 shows the histograms of averaged cortical thickness maps for each brain volume ( 3 for subject 1 and 1 each for subjects 2 and 3). With sulcal segmentation (solid lines), all of the histograms show a peak between 2-3 mm, which is consistent with results in the literature [7-10]. The within subject results (Fig. 5a) show good agreement with each other. The literature states that cortical thickness measures up to $5 \mathrm{~mm}$, but our histograms show some values greater than this. We attribute these large values to sub-cortical grey matter structures. Without sulcal segmentation (dashed lines in Fig 5), thickness values are significantly greater.

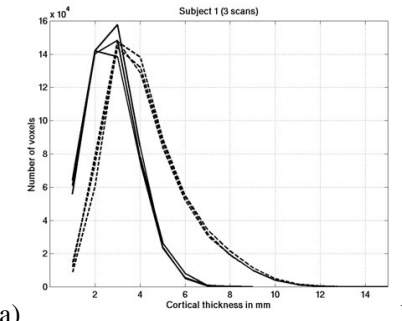

a)

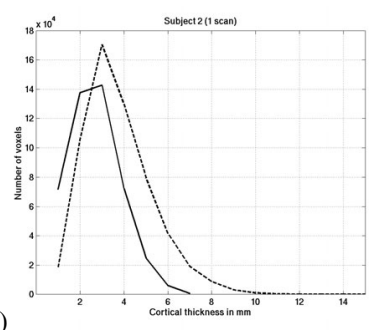

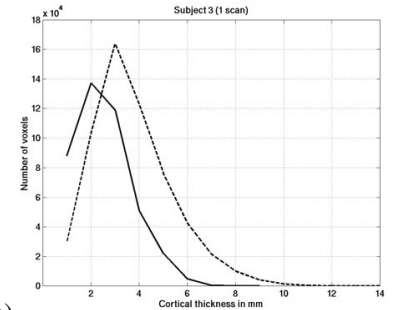

c)

Fig. 5. Histograms of whole brain cortical thickness measurements (in $\mathrm{mm}$ ) for three subjects (a-c), with (solid lines) and without (dashed lines) sulcal segmentation. Subject 1 (a) has 3 sets of results

Surface Rendering of Cortical Thickness Maps. Figure 6 shows surface renderings of the averaged cortical thickness maps for the left and right hemisphere of each sub- 
ject. Regions where the surface renderings show thinner cortex (darker areas) correspond to regions of the brain where the cortex is known to be thinner [2]. This can be seen clearly at the back of the brain (occipital lobe) in all subjects and in the top central part of the brain (central sulcus) in the first and third subjects. The standard deviation of the thickness maps calculated for subject 1 (Fig. 6b) is less than $1 \mathrm{~mm}$ and tends to be larger where the cortex is wider. It must be noted that the standard deviation also includes any small errors that arise from matching the images.

a)
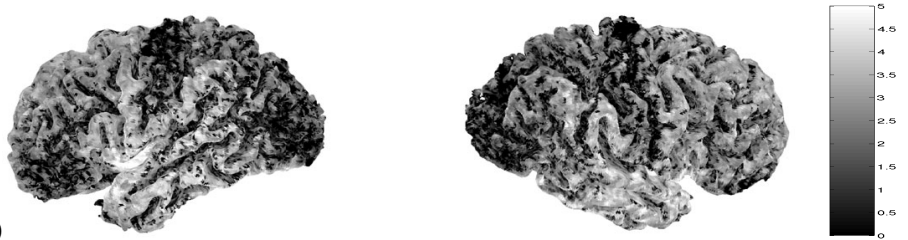

b)


c)
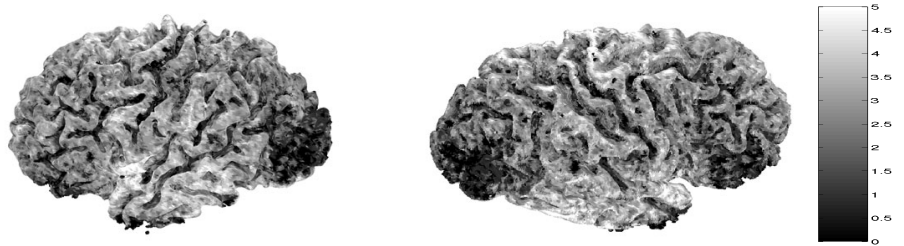

d)


Fig. 6. Surface renderings of averaged cortical thickness maps. a) Mean of 3 coregistered averaged thickness maps for subject 1. b) Standard deviation of 3 coregistered averaged thickness maps for subject 1. c) and d) Averaged thickness maps for subjects 2 and 3. The shading of each surface represents the local thickness in $\mathrm{mm}$ corresponding to the grey scale bar. For visualization purposes, the renderings have been thresholded at a thickness of $5 \mathrm{~mm}$ to give an optimal intensity range

\section{Summary and Conclusions}

We present a method to segment deep sulci for cortical thickness measurements. The method divides the cortex into sub-layers and uses the sub-layer thicknesses to determine the location of sulci. Laplace's equation is solved for each sub-layer to correctly determine its thickness. Our results are consistent with the literature and also show good agreement within subject. 
Our approach for segmenting sulci can be considered as an automatic feature detection method that exploits the laminar structure of the cortex. In this context, our method could also provide efficient feature detection based on the underlying neuroanatomy for deformable surface models.

In this work have used the original MRI resolution of $1 \mathrm{~mm}$ for all processing steps. However, using interpolation, our methods can be adapted to use a smaller sampling size with the disadvantage of increased processing times. The robustness of the morphological operations used to construct the final grey matter CSF border and the accuracy of the thickness measurements may be dependent on this sampling size. Therefore a full analysis of the errors related to the sampling resolution is required. This is a topic of our current work, as well as a more extensive validation using a larger number of subjects, regional analyses of cortical thickness and comparison with results using a previously published method.

This work was funded by the Wellcome Trust, London, UK.

\section{References}

1. Geyer, S., Schleicher, A., Zilles, K.: Areas 3a, 3b, and 1 of Human Primary Somatosensory Cortex 1. Neuroimage 10 (1999) 63-83.

2. Meyer, J.R., Roychowdhury, S., Russell, E.J., Callahan, C., Gitelman, D., Mesulam, M.M.: Location of the central sulcus via cortical thickness of the precentral and postcentral gyri on MR. AJNR Am J Neuroradiol. 17 (1996) 1699-706.

3. Rusinek, H., de Leon, M.J., George, A.E., Stylopoulos, L.A., Chandra, R., Smith, G., Rand, T., Mourino, M., Kowalski, H. Alzheimer disease: measuring loss of cerebral gray matter with MR imaging Radiology 178 (1991) 109-114.

4. Terry, R.D., Peck, A., DeTeresa, R., Schecter, R., Horoupian, D.S.: Some morphometric aspects of the brain senile dementia of the Alzheimer type. An.of Neuro.10(1981)184-192.

5. Stokking, R., Vincken, K.L., Viergever, M.A.: Automatic morphology-based brain segmentation (mbrase) from mri-T1 data. Neuroimage 12 (2000) 726-738.

6. Deichmann, R., Good, C.D., Josephs, O., Ashburner, J., Turner, R.: Optimization of 3-D MP-RAGE sequences for structural brain imaging. NeuroImage 12 (2000) 112-127.

7. Kruggel, F., Yves-von-Cramon, D.:Measuring the cortical thickness [MRI segmentation procedure] Proceedings IEEE Workshop on Mathematical Methods in Biomedical Image Analysis. IEEE Comput. Soc, Los Alamitos, CA, USA; (2000) 154-61.

8. MacDonald, D., Kabani, N., Avis, D., Evans, A.C.: Automated 3-D extraction of inner and outer surfaces of cerebral cortex from MRI. Neuroimage 12 (2000) 340-356.

9. Fischl, B., Dale, A..M.:Measuring the thickness of the human cerebral cortex from magnetic resonance images. Proc Natl Acad Sci U S A. 97 (2000) 11050-5.

10. Jones, S.E., Buchbinder, B.R., Aharon, I.: Three-Dimensional Mapping of Cortical Thickness Using Laplace's Equation. Human Brain Mapping 11 (2000) 12-32.

11. Ashburner, J., Friston, K.: Multimodal Image Coregistration and Partitioning-A Unified Framework. Neuroimage 6 (1997) 209-217.

12. Matlab 5 and Matlab 6 Copyright 1984-2000 The MathWorks, Inc. Natick, MA, USA. 